\section{Does the urban environment cause psychosis? ${ }^{\dagger}$}

\author{
JIM VAN OS
}

The paper by Sundquist $e$ t al is a welcome addition to a growing body of evidence linking exposures in the urban environment to the onset of schizophrenia (Sundquist et al, 2004, this issue). They also report, in agreement with the literature, that a similar association exists for depression of severity requiring hospital admission, albeit of a much lower effect size than that for schizophrenia and with the caveat that only in a small and biased proportion of cases is the person with clinical depression ever admitted to hospital. The paper represents a truly prospective analysis, and also considers confounding by other important demographic variables and changing exposure status over the period of observation.

The fact that the incidence of schizophrenia increases consistently with increasing levels of urbanicity in a dose-response fashion suggests not only statistical association, but also causality. Thus, the Swedish findings, in combination with earlier publications, allow us to put forward an increasingly plausible case that the environment has a powerful influence on variation in the incidence of schizophrenia in populations. The identification of the nature of this environmental exposure is likely to further significantly our knowledge of the causes and mechanisms that facilitate symptom formation in psychosis.

\section{SCIENTIFIC VALIDITY, CONCEPTUAL UNCERTAINTY}

Compared with earlier environmental candidates for a causal role in psychosis, such as prenatal influenza, prenatal and perinatal complications, early family environment and life events, the urban exposure is relatively untainted in terms of

†See pp. 293-298, this issue. inconsistencies in the findings, failure to replicate, major biases inherent to research designs and heated debates about exposure measurement. In fact, the production of a string of consistent and methodologically sound research reports on urbanicity and schizophrenia from a variety of countries and settings appears to have been so rapid that their full significance may require some time to sink in. One of the reasons for the relative lack of urgency in responding to the consistent epidemiological finding that several studies suggest might explain more than $30 \%$ of all schizophrenia incidence is also its main conceptual weakness: there is no valid explanation as to what constitutes the true nature of the environmental exposure that poses as 'urbanicity'.

\section{A WIDESPREAD EXPOSURE WITH WIDESPREAD EFFECTS}

It has been shown that intra- or intergenerational drift or other forms of spatial selective mobility are unlikely to be the main factor explaining the high rates of psychosis in urban areas (Lewis et al, 1992; Pedersen \& Mortensen, 2001a,b). There is general agreement that the kind of geographical variation in incidence associated with urbanisation is indicative of an environmental effect (although not excluding a contribution of genetic effects - see below), and that this effect has its impact, through continuous or repeated exposure, on developing children and adolescents that is, the time window of exposure is not around the time of birth and not around the time of onset of psychotic disorder, but in between (Marcelis et al, 1999; Pedersen \& Mortensen, 2001a). Recent studies in Germany, Greece, the UK and The Netherlands (e.g. van Os et al, 2001; Stefanis et al, 2004) have shown that the increased level of risk of psychotic disorder in urban populations is not phenotypically silent, because the prevalence of at-risk mental states characterised by subtle psychosis-like phenomena is also higher in urban areas, independent of the increased rate of psychotic disorder, and independent of service use and socio-demographic factors including ethnic group, drug use and size of social network (Pedersen $\&$ Mortensen, 2001a,b; van Os et al, 2001). Recent analyses also suggest that the increased risk for clinical and non-clinical expressions of psychosis in urban areas is not mediated by neuropsychological impairment, obstetric complications or childhood socioeconomic position (Harrison et al, 2003; Stefanis et al, 2004). Although viral hypotheses have been put forward to explain the effect of urbanicity, household crowding is not a risk factor for schizophrenia and a hypothetical viral exposure for a rare disorder is arguably difficult to reconcile with the absence of a mediating effect of cognitive variables and with elevated prevalences of subtle psychosis-like experiences of $23 \%$ in the most urban areas $v .13 \%$ in the most rural areas (van Os et al, 2001). The high prevalence of such at-risk mental states suggests a much more widespread exposure with a cumulative effect over the course of development.

\section{CLUES TO THE NATURE OF THE EXPOSURE}

The environmental exposure in urban areas thus appears to affect individuals by shaping an enduring and phenotypically at least partly detectable liability to psychosis, through continuous or repeated exposure in childhood and adolescence prior to the onset of psychosis. This effect is at least in part specific for psychosis, because associations with incidence of affective disorder are much weaker. For this reason, a generic effect of stress is unlikely to explain the entire association between schizophrenia and urbanicity, although a part of the aetiology that is shared with depression may be related to the impact of the stresses of urban life. What constitutes urban stress has yet to be validated, however, although stress due to noise is a possible candidate. Direct effects of pollution have been hypothesised but have little biological plausibility. Differences in health behaviours may play a part, but substance misuse has been widely controlled for in studies of the association between schizophrenia and urbanicity. Remarkably little attention has been paid to social factors. Recently, 
however, aspects of the wider social environment have been shown to affect mental health outcomes, also in relation to psychosis. For example, aspects of the wider social environment such as community level of social fragmentation, social isolation and social inequality may account for variation in the incidence of schizophrenia in small areas (van Os et al, 2000). The impact of such variables may be truly ecological, affecting every person in the community regardless of their individual socio-demographic and other characteristics, thus creating a distributed liability in exposed populations. Although most of the studies focusing on such ecological exposures involved comparisons between small areas such as neighbourhoods, it is plausible that similar mechanisms operate to explain the differences between urban and rural environments.

\section{URBANICITY AND GENETIC LIABILITY}

In order to explain the hypothetical relationship between an area-level exposure such as social fragmentation and an individual-level outcome such as psychotic symptoms, individual psychological mechanisms must be invoked. For example, individuals with a genetic liability to schizophrenia might be less likely to have their early abnormal mental states corrected in urban areas with higher levels of social fragmentation or lower levels of informal social control, resulting in higher rates of expression of psychosis. Similarly, aspects of the wider social environment such as lack of perceived safety or social stress might directly contribute to the development of cognitive vulnerabilities for psychosis, resulting in higher rates of psychotic disorder

JIM VAN OS, Department of Psychiatry and Neuropsychology, South Limburg Mental Health Research Network, EURON, Maastricht University, PO Box 616 (DRT I0), 6200 MD Maastricht, The Netherlands. Tel: +3l 43 3875443; fax: +3l 43 3875444; e-mail: j.vanos@sp.unimaas.nl; and Division of Psychological Medicine, Institute of Psychiatry, London, UK

(First received 26 September 2003, accepted 12 October 2003)

in the genetically predisposed. A general mechanism of gene-environment interaction is supported by evidence suggesting synergism between urbanicity and familial liability in their effect on psychotic disorder (van Os et al, 2003).

\section{CONCLUSIONS}

The nationwide Swedish study corroborates and adds to earlier evidence that an environmental exposure operates in urban areas to increase the prevalence of at-risk mental states and the incidence of psychotic disorder. The impact of this exposure is substantial, although it may well reflect a joint effect of urban environmental exposure and other (possibly genetic) factors. The role of the wider social environment and its ecological effect on the development in young people of cognitive vulnerabilities for psychosis has been neglected as a possible mechanism. If there are environmental causes for psychosis, urban ecological studies are likely to help provide new insights in the years to come.

\section{DECLARATION OF INTEREST}

None.

\section{REFERENCES}

Harrison, G., Fouskadis, D., Kasmussen, F., et al (2003) Association between psychotic disorder and urban place of birth is not mediated by obstetric complications and childhood socio-economic position: a cohort study. Psychological Medicine, 33, 723-731.

Lewis, G., David, A., Andreasson, S., et al (1992) Schizophrenia and city life. Lancet, 340, 137-140.

Marcelis, M., Takei, N. \& van Os, J. (1999)

Urbanization and risk for schizophrenia: does the effect operate before or around the time of illness onset? Psychological Medicine, 29, 1197-1203.

Pedersen, C. B. \& Mortensen, P. B. (200la) Evidence of a dose-response relationship between urbanicity during upbringing and schizophrenia risk. Archives of General Psychiatry, 58, 1039-1046.

Pedersen, C. B. \& Mortensen, P. B. (200lb) Family history, place and season of birth as risk factors for schizophrenia in Denmark: a replication and reanalysis. British Journal of Psychiatry, 179, 46-52.

Stefanis, N. C., Delespaul, P., Smirnis, N. K., et al (2004) Is the excess risk of psychosis in urban areas attributable to altered cognitive development? Social Psychiatry and Psychiatric Epidemiology, in press.

Sundquist, K., Frank, G. \& Sundquist, J. (2004) Urbanisation and incidence of psychosis and depression. Follow-up study of 4.4 million women and men in Sweden. British Journal of Psychiatry, 184, 293-298.

Van Os, J., Driessen, G., Gunther, N., et al (2000) Neighbourhood variation in incidence of schizophrenia. Evidence for person-environment interaction. British Journal of Psychiatry, 176, 243-248.

Van Os, J., Hanssen, M., Bijl, R. V., et al (200I) Prevalence of psychotic disorder and community level of psychotic symptoms: an urban-rural comparison. Archives of General Psychiatry, 58, 663-668.

Van Os, J., Hanssen, M., Bak, M., et al (2003) Do urbanicity and familial liability coparticipate in causing psychosis? American Journal of Psychiatry, 160, 477-482. 